\title{
Genetic mechanism of ASD-related monogenetic diseases
}

\author{
Yuqing Chen ${ }^{1, *, \dagger}$, and Yasong Pang ${ }^{2, \dagger}$ \\ ${ }^{1}$ School of International Pharmaceutical Business, China Pharmaceutical University, Nanjing, Jiangsu Province, 211198, China \\ ${ }^{2}$ School of Biochemistry and Cell Biology, University College Cork, Cork, Co Cork, Ireland T12 K8AF \\ †These authors contribute equally.
}

\begin{abstract}
Autism spectrum disorder (ASD) is a series of neurodevelopmental disorders presented as behavioral abnormality such as problems with social, communication, and repetitive behaviors. There are four ASD-related monogenetic diseases worthy of analysis: fragile X syndrome, PTEN hamartoma tumor syndrome, tuberous sclerosis complex, and Rett syndrome. These four monogenetic diseases are respectively caused by one gene and have something in common in phenotype while also have their unique features. All of them have been related to the same pathway: phosphatidylinositol 3-phosphate kinase (PI3K) / protein kinase $\mathrm{B}$ (PKB or Akt) / mammalian target of rapamycin (mTOR) signaling pathway, which plays a primary role in many physiological processes on a cellular level. Nowadays, there is no cure for these monogenetic diseases, thus much remains to be done to find ways to treat patients with the diseases mentioned above.
\end{abstract}

\section{Introduction}

Autism spectrum disorder (ASD) is a term to describe a series of formidable polygenic diseases for neuroscience and clinical therapeutics, due to its substantial heterogeneity, lifelong nature, high prevalence, and complexity [1]. The clinical symptoms of ASD include early appearing social communication deficits and repetitive sensory-motor behaviors [2]. The autistic symptoms commonly appear during the period of infancy or childhood. According to the newest data from CDC (Centers for Disease Control and Prevention) [3], approximately one in 54 children has been diagnosed with ASD in the United States, and the data grows dramatically year by year. Unfortunately, there is no treatment to completely cure ASD, but nowadays researchers provided some feasible theoretical bases for gene therapy of ASD.

ASD is associated with a combination of genetic and environmental factors. One of the most common environmental factors is unhealthy eating habits during pregnancy. Although the genetic factors for ASD are still unclear yet, researchers have found candidate susceptibility loci in recent decades and have established brain imaging data for analysis. Chromosomal copy number variants (CNVs) are mainly known to contribute to neurodevelopmental disorders including ASD. On the other hand, it is confirmed ASD can affect information processing in the brain, as well as the connection and organization of nerve cells and synapses.

In this review, we focused on the genetic mechanism of four ASD-related monogenetic diseases: fragile $\mathrm{X}$ syndrome (FXS), PTEN hamartoma tumor syndrome (PHTS), tuberous sclerosis (TSC), and RTT, and

*Corresponding author. Email: 2020200457@stu.cpu.edu.cn overviewed a common molecular pathway among the four diseases.

\section{Fragile X Syndrome (FXS)}

FXS is an inherited X-linked disorder causing emotional and intellectual impairments. The prevalence of FXS with a full mutation is assessed at 1 in 4,000 in males and 1 in 5,000 to 8,000 in females [4], with the difference being because FXS is a commonly sex-linked disease. This section will discuss the FXS-related gene and FXS-ASD comorbidities.

\subsection{FXS-related gene}

The transcriptional silencing of the fragile $\mathrm{X}$ mental retardation 1 (FMRI) gene and the loss of its protein product, fragile $\mathrm{X}$ mental retardation protein (FMRP), is considered to be the only genetic factor to cause FXS. FMRI is highly conserved, located on the long arm of the $\mathrm{X}$ chromosome, and widely expressed in early development. FMRP mainly can be detected in the brain, gonads, and lymphocytes in this period of growth. In terms of human neurodevelopment, evidence suspects that an NLGN3/CYFIP/FMR1-centered synaptic pathway plays a role in the phenotypes linked to the physiological and structural plasticity features of ASD [5]. NLGN3 (neuroligin 3), CYFIP (cytoplasmic FMR1 interacting protein), and FMR1 are involved in dendritic spine morphology relevant to ASD. Furthermore, a new sequencing strategy is used to confirm FMR1 targets in neural progenitors of the human ventral and dorsal forebrain [6]. 
Expansion of unstable trinucleotide (CGG) repeats near the 5' end of FMR1 as well as within an untranslated promoter region is always the only mutation underlying FXS. The amplification of the repeated CGG region varies between individuals, leading to the methylation of upstream $\mathrm{CpG}$ islands, the prevention of gene expression, and finally changing the phenotype of FXS [7]. In animal knockout (KO) trials, the FMR1-loss mice show a series of ASD symptoms, such as perseverative behaviors, inappropriate social interactions, and learning and memory defects [8]. In the latest FMR1 KO experiment, D'Antoni's study has reckoned mitochondrial respiratory chain complexes are applied as an alteration of mitochondrial oxidative phosphorylation (OXPHOS) in the cerebral cortex [9]. Together, both studies illustrated the FMR1 loss-of-function would drive neuronal deficiencies to a differing extent.

FMRP is a polyribosome-related neuronal RNA-binding protein, acting in inhibiting the translation of specific mRNAs at synapses, mainly associating with ribonucleoparticles and multiple metabolic pathways. A clue for the role of FMRP was provided by the structure of FMRP, covering two types of RNA-binding motifs: two K homology $(\mathrm{KH})$ domains and one RGG box region. The great majority of FMRP binds to the coding sequence (CDS), relating to some transcripts where ribosomes stall during translation [10]. Loss of FMRP causes impaired synaptic plasticity, human cognitive deficits and other autistic features.

\subsection{ASD comorbidity in FXS}

The patients who suffer from FXS, also experience co-occurring behavioral defects including ASD symptoms [11]. There are several factors linked to ASD symptoms within FXS. The ASD symptoms appear in one-year-old children with FXS, which remain stable until the child is 5 years old, with ASD symptoms noticeably changing once the children progress to school years [12]. Evidence shows the reduction of social avoidance behaviors for males with FXS from early childhood to mid-adolescence [13]. Also, the cognitive ability for patients with comorbid ASD and FXS is lower than those with FXS without ASD [14]. With regards to language ability, the relationship to ASD within FXS varies among different dimensions of language [14]. From a molecular view, FMRP is responsible for regulating several genes that are closely related to ASD such as the SHANK family and mTOR, and it indirectly affects ASD symptoms [15].

\section{PTEN Hamartoma Tumor Syndrome (PHTS)}

PHTS is a type of spectrum disorders due to the mutations of PTEN, a typical tumor suppressor gene in germ cells. The subdivisions of PHTS include Cowden syndrome (CS), Bannayan-Riley-Ruvalcaba syndrome (BRRS), Proteus syndrome, Proteus-like syndrome, and ASD with macrocephaly [16]. The primary symptoms in
PHTS include increased risk for certain types of cancer such as glioblastoma and breast cancer, tumor-like malformations like hamartomas [17], and neurodevelopmental disorders such as ASD. Due to the subtle external manifestations within CS and BBRS, the prevalence of PHTS is hard to define. According to a general estimate, however, the prevalence is about 1 in 200,000 [18], which is certainly lower than in actual clinical situations. This section overviews the candidate gene of PHTS and the animal models for the PTEN-ASD study.

\subsection{PTEN}

PHTS is one of the monogenic disorders in ASD caused by the mutations of PTEN. These 'alterations' are on human chromosome 10 and all or part of PTEN would be deleted in some cases. Most of them happen in the promoter region, and some are intragenic and missense mutations [19]. The phosphatase protein product of PTEN is a vital element in antagonizing the $\mathrm{PI}$ KK/Akt/mTOR pathway to regulate the cell cycle (See Section 5) [20]. Looking at the mechanisms of this pathway, it is confirmed that loss of PTEN in humans not only results in the development of various cancers, but seriously affects neural proliferation, survival, synaptic connectivity, and other neurodevelopmental processes [21]. Furthermore, many studies reported some individuals carrying PTEN mutations suffered from both PHTS and ASD [22], indicating PTEN acts as a risk factor in ASD.

\subsection{PTEN Murine Models}

Although it is fully understood how PTEN regulates molecular signaling in tumor cell lines, we only know little about the influence of PTEN on brain physiology and behaviors. Therefore, plenty of animal models, primarily murine models, are generated to further explore the function of PTEN in ASD and neurogenesis.

PTEN homologous KO mice are embryonic lethal [23], and heterozygous mice possess overly large brains similar to the macrocephaly occurring in individuals with ASD and PTEN mutations [24, 25]. Accelerated and enlarged growth in specific brain regions during development is a hallmark of ASD patients [26], although the connection between an abnormal brain and the core symptoms of ASD is not yet clear enough. Collectively considered with other relevant PTEN transgenic reports, PTEN mutant is involved in both different cancer types and neuronal defects associated with ASD.

To discover the association of PTEN localization with brain development, the $\mathrm{m} 3 \mathrm{~m} 4$ mutant strain is applied as a constitutive knock-in system to restrict PTEN predominantly to the cytoplasm and its global expression with time, which contains five-point variants in PTEN's C2 domain [21, 27]. The level of PTEN is reduced in homozygous PTENm3m4 mice including a dramatic decline in nuclear and region-specific increases [28]. The cytoplasm-prepotent protein leads to cellular hypertrophy 
but it is restricted to the soma. In contrast, PTEN would rescue neuronal hypertrophy in wild-type mice [29]. Moreover, this protein contributes to repressing neuronal differentiation, maturation, and other disorders relevant to PTEN-ASD phenotypes. Therefore, the PTENm3m4 mutation can be a useful model for deep research about PTEN-ASD behaviors, even if more comprehensive studies are required to overcome its shortcomings.

\section{Tuberous Sclerosis Complex (TSC)}

Tuberous sclerosis complex (TSC) is an autosomal dominant genetic disease that influences not only the brain but also many other organs throughout the human body. Nearly $80 \%$ of patients with TSC have epilepsy, with about two-thirds of the cases having severe mental retardation. Also, there are obvious behavioral problems such as seizures, infantile spasms, and sleep disturbance at a young age [30]. The prevalence of TSC is about 7 to 12 per 100,000 [31].

\subsection{Candidate gene}

Molecular testing indicates that mutations in gene TSC1 and TSC2 are the major reason lying behind TSC, for about $75-90 \%$ of TSC patients carry mutations relevant to these two genes [32]. Both TSC1 and TSC2 are tumor suppressor genes, whose translating product is mTORC1 (mammalian target of rapamycin complex 1). TSC1 is located on chromosome 9 q34 with 21 exons, whereas TSC2 is located on chromosome 16 p13.3 with 41 exons [33]. Cases related to the two genes are not actually the same. Patients with a TSC2 mutation are more than those with a TSC1 mutation, even two to ten times the latter, with a more severe series of syndromes [34]. On the other hand, those with a TSC1 mutation are more probable to be familial hereditary.

TSC1 and TSC2 encode the protein hamartin and tuberin respectively, whose combination is the tumor suppressor, mTORC1. This protein complex participates in the mTOR pathway, functioning as a significant way to mediate cellular activity [33]. In addition, the PI3K signaling pathway fundamentally relies on TSC1 and TSC2. The downstream effectors act as an activator of the GTPase activity of Ras homolog enriched in the brain (RHEB) [35].

As mentioned above, the proper operation of these signaling pathways is determined by the correct function of TSC1 and TSC2. Different mutations may lead to various problems. While patients have a large genomic deletion, there will be protein synthesis prevention. In other cases, those who have a nonsense mutation will clearly have their TSC1 or TSC2 proteins inactivated [33].

\subsection{Recent research}

To make out the relationship between some typical syndromes such as epilepsy or seizure and early development in TSC, researchers evaluated 130 infants and children with TSC in a prospective and longitudinal way. This analysis reinforces the great negative influence that seizure and epilepsy exert on normal neurodevelopment [36]. It is important to highlight the fact that early diagnosis of TSC should be paid attention to so that TSC development during infancy could be controlled through early treatment.

\section{Rett syndrome (RTT)}

Rett syndrome, also known as RTT, is a well-known neurodevelopmental disorder, which is characterized by a rapid regression at the age of 6 to 18 months, including speech losses and motor disorders such as repetitive and stereotypic hand movements, hypotonia, epilepsy, and breathing irregularities [37]. RTT is a typical kind of ASD with an incidence of about $1 / 8500$ in females.

RTT has an obvious difference between males and females. It mostly influences females since the gene MECP2 talked about later is located on chromosome Xq28. But it does not mean that males would not be affected by RTT. Actually, similar variants have been viewed in male patients, which is caused by duplication of MECP2 [38].

\subsection{Candidate gene}

The most important candidate gene lying behind RTT is MECP2, with 95\% RTT patients have MECP2 mutation. The important role of MECP2 in RTT has been proofed by animal models. It is noticeable that not all animal models show obvious features of autism, which depends on individual differences and its combination with $\mathrm{X}$ chromosome inactivity (XCI) [39]. Besides MECP2, there are also variants of RTT caused by mutations in genes such as CDKL5, FOXG1 and MEF2C, which only account for the minority of RTT patients [7].

As for the MECP2 protein, which is encoded by the MECP2 gene, consists of several domains. The combination of Minimal Brain Dysfunction and Treatment-Resistant Depression can associate with chromatin factors such as histone deacetylase inhibitor (HDAC), Yin and Yang 1 protein (YY1), Recombinant DNA Methyltransferase 1 (DNMT1). The connective tissue disease (CTD) is viewed as necessary for the protein's integrity, containing a WW-domain-binding region. The domains mentioned above are prone to mutations, thus causing RTT or similar autism. Neural tube defects (NTD) are not so much referred to as the others, but it is crucial to the differential between MECP2-e1 and MECP2-e2, two main isoforms of the protein MECP2. The effect of MECP2 phosphorylation is of great importance in the activation of pS421 and pS80, which are primarily participated in neuronal maturation and activity-dependent neuronal response [7].

Over $70 \%$ of mutations in MECP2 lie in the third and the fourth exons, which are known as MBD and TRD domains. The mechanism of the mutations is various. For example, a novel mutation in exon 3, p. Gly119Gly variant may create an aberrant splice site, though the nucleotide mutation will not lead to the coded amino acid 
change. Despite that, mutations in exon 4 are also common, within which there is a hotspot for deletion occurrence. Both of the two novel mutations will give rise to the frameshift of the protein MECP2, thus influencing the proper function of the protein [40].

\subsection{Recent research}

Researchers have been underway to understand more about RTT. Recently, a study showed that a female mouse model can demonstrate typical behaviors of RTT better than its male offspring. Moreover, it indicated that RTT is also influenced by the rearing conditions [41]. Another study showed that germline mutations in MECP2 are the cause underlying most RTT cases. Such kind of mutation will lead to obvious autism symptoms in females while death in males before the age of 2 [42]. This implies that while faced with RTT patients of different genders, the medical staff should choose various treatments on purpose.

\section{Common pathway}

One emerging pathway common to all four syndromic
ASD disorders is the $\mathrm{PI} 3 \mathrm{~K} / \mathrm{Akt} / \mathrm{mTOR}$ pathway.

\subsection{Introduction}

Concerning the PI3K/Akt/mTOR pathway, there are three pathways while they can interact with each other and collaborate to regulate the body. The name of each pathway indicates the core protein engaged in it. The first one is PI3K, which is a phosphatidylinositol kinase and serine-threonine protein kinase itself. The product of this pathway, PI3P, activates cell trafficking. Additionally, PI $(3,4)$ P2 activates cell growth and protects cells from apoptosis. Following is Akt, also called PKB or Rac, known as protein kinase $\mathrm{B}$. The phosphorylation of Akt through the Akt pathway also makes cell growth activation realizable. The last one is mTOR, known as the mammalian target of rapamycin. The mTOR pathway is related to normal cell metabolism and the tumor. These pathways can not only work separately but also combine with each other.

Overall, the PI3K/Akt/mTOR pathway plays an important role in many cellular processes including cell growth, proliferation, and differentiation. As shown in Figure 1, the process of the whole pathway is complex.

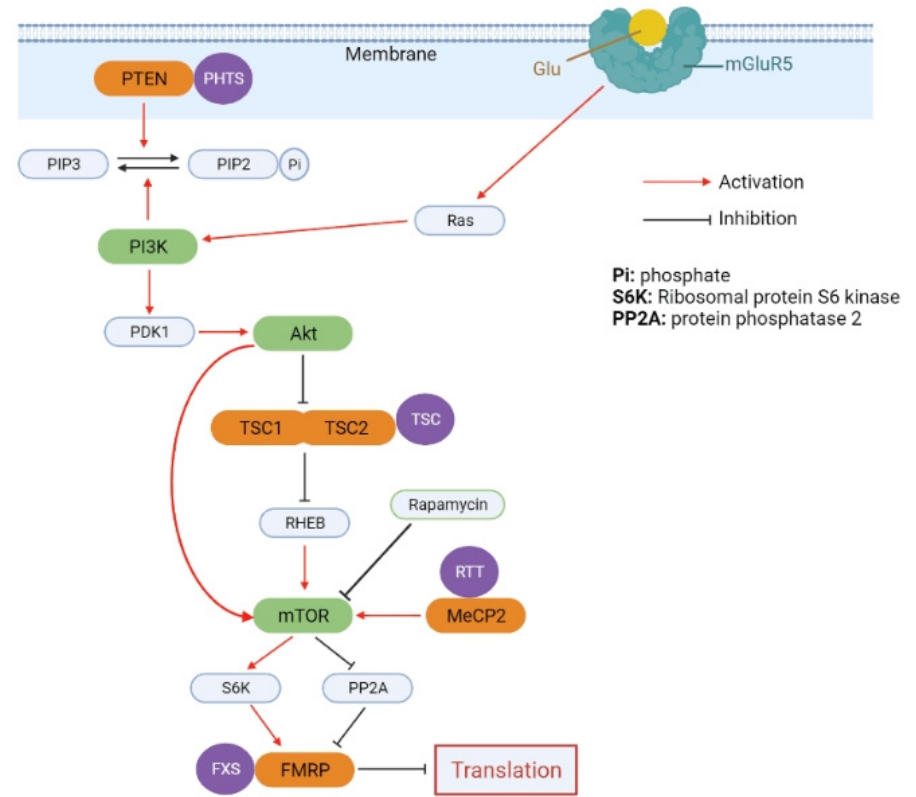

Fig. 1. The PI3K/Akt/mTOR signaling pathway.

Schematic diagram illustrating how four genes get involved in the PI3K/Akt/mTOR pathway, the major components of the pathway, their interactions, and downstream cellular functions. Signaling molecules that are involved in ASD are highlighted in orange, and the related monogenic disorders are highlighted in purple.

The PH domain of AKT can be attached to the downstream product of PI3K, PI $(4,5)$ P2 and PI $(3,4,5)$ $\mathrm{P} 3$, thus being activated [43]. mTOR relies on the heterodimer of TSC1 and TSC2 protein, which can be promoted by AKT. Therefore, the three pathways are combined and do their part to adjust the physiological process [43].

\subsection{Relationship between the common pathway and the genes of four ASD-related disorders}

PTEN is a lipid phosphatase located in all human cell types. The best-characterized role of PTEN is to dephosphorylate

(3,4,5)-trisphosphate (PIP3), producing phosphatidylinositol (4,5)-biphosphate PIP2, and directly inhibiting the Akt pathway [44]. In contrast, class I PI3K contributes to activate phosphoinositide-dependent kinase 1 (PDK1, which then increases the activation of Akt), and convert PIP2 to PIP3, so PI3K and PTEN are a pair of antagonistic enzymes. Followed by the PTEN-PI3K pathway, Akt would play a role in 
negatively regulating $\mathrm{TSC} 1 \& 2$, as well as activating mTOR [7]. TSC1\&2 works as a repressor for RHEB, which will also activate the mTOR pathway [7]. In terms of FMRP, it binds and prevents the translation of some mRNAs, including metabotropic glutamate receptor 5 (mGluR5) and members in the PI3K/Akt/mTOR pathway [45]. mGluRs are involved in synaptic plasticity via the PI3K/Akt/mTOR pathway. Put simply, FMRP, PTEN, TSC1\&2 act as a suppressor in the PI3K/Akt/mTOR pathway.

As for MECP2, a different pattern has been displayed in the PI3K/Akt/mTOR pathway. From Ehrhart's paper, MECP2 shows effects on translation processes, especially protein synthesis, by enhancing the Akt/mTOR pathway [46]. Collectively, the genes causing the monogenic ASD-related disorders play diverse but critical roles in the $\mathrm{PI} 3 \mathrm{~K} / \mathrm{Akt} / \mathrm{mTOR}$ pathway.

\section{Conclusion}

So far, an increasing number of discoveries about the genetic factors of ASD have been reported for several decades. However, ASD is still a global and unsolved challenge for humans. With further research, it was found that sometimes ASD may co-occur with four monogenic disorders: FXS, PHTS, TSC, and RTT, caused by the changes in the FMR1, PTEN, TSC1/TSC2, and MECP2 genes respectively. These genes mainly influence neurodevelopment and synaptic plasticity, resulting in impaired cognitive ability and other ASD-related phenotypes. Additionally, all of them function in the PI3K/Akt/mTOR pathway which contains several core steps in regulating cell behaviors such as cell growth and cell survival. Similar to ASD, there is no cure for monogenic disorders, thus more experiments and more animal trials are required to find the possibility of a cure. Perhaps autism and its comorbid diseases can be cured soon with the efforts of scientists.

\section{References:}

1. Di Martino, A., et al., The autism brain imaging data exchange: towards a large-scale evaluation of the intrinsic brain architecture in autism. Molecular Psychiatry, (2014). 19(6): p. 659-667.

2. Lord, C., et al., Autism spectrum disorder. The Lancet, (2018). 392(10146): p. 508-520.

3. Maenner, M.J., K.A. Shaw, and J. Baio, Prevalence of autism spectrum disorder among children aged 8 years-autism and developmental disabilities monitoring network, 11 sites, United States, 2016. MMWR Surveillance Summaries, (2020). 69(4): p. 1.

4. Niu, M., et al., Fragile X syndrome: prevalence, treatment, and prevention in China. Frontiers in neurology, (2017). 8: p. 254.

5. Sledziowska, M., J. Galloway, and S.J. Baudouin, Evidence for a contribution of the Nlgn3/Cyfip1/Fmr1 pathway in the pathophysiology of autism spectrum disorders. Neuroscience, (2020). 445: p. 31-41.

6. Li, M., et al., Identification of FMR1-regulated molecular networks in human neurodevelopment. Genome research, (2020). 30(3): p. 361-374.

7. Buxbaum, J.D. and P.R. Hof, The neuroscience of autism spectrum disorders. (2012): Academic Press.

8. Bernardet, M. and W.E. Crusio, Fmr1 KO mice as a possible model of autistic features. TheScientificWorldJOURNAL, (2006). 6: p. 1164-1176.

9. D'Antoni, S., et al., Aberrant mitochondrial bioenergetics in the cerebral cortex of the Fmrl knockout mouse model of fragile $\mathrm{X}$ syndrome. Biological chemistry, (2020). 401(4): p. 497-503.

10. Darnell, J.C., et al., FMRP stalls ribosomal translocation on mRNAs linked to synaptic function and autism. Cell, (2011). 146(2): p. 247-261.

11. Budimirovic, D.B. and W.E. Kaufmann, What can we learn about autism from studying fragile $\mathrm{X}$ syndrome? Developmental neuroscience, (2011). 33(5): p. 379-394.

12. Roberts, J.E., et al., Heart activity and autistic behavior in infants and toddlers with fragile $\mathrm{X}$ syndrome. American journal on intellectual and developmental disabilities, (2012). 117(2): p. 90-102.

13. McDuffie, A., et al., Autism spectrum disorder in children and adolescents with fragile $\mathrm{X}$ syndrome: within-syndrome differences and age-related changes. American Journal on Intellectual and Developmental Disabilities, (2010). 115(4): p. 307-326.

14. Abbeduto, L., et al., ASD comorbidity in fragile X syndrome: Symptom profile and predictors of symptom severity in adolescent and young adult males. Journal of autism and developmental disorders, (2019). 49(3): p. 960-977.

15. Niu, M., et al., Autism symptoms in fragile $X$ syndrome. Journal of child neurology, (2017). 32(10): p. 903-909.

16. Pilarski, R., PTEN hamartoma tumor syndrome: a clinical overview. Cancers, (2019). 11(6): p. 844.

17. Mester, J. and C. Eng, PTEN hamartoma tumor syndrome. Handbook of clinical neurology, (2015). 132: p. 129-137.

18. Nelen, M.R., et al., Novel PTEN mutations in patients with Cowden disease: absence of clear genotype-phenotype correlations. European Journal of Human Genetics, (1999). 7(3): p. 267-273.

19. Buxbaum, J.D., et al., Mutation screening of the PTEN gene in patients with autism spectrum disorders and macrocephaly. American Journal of Medical Genetics Part B: Neuropsychiatric Genetics, (2007). 144(4): p. 484-491.

20. Rademacher, S. and B.J. Eickholt, PTEN in autism and neurodevelopmental disorders. Cold Spring 
Harbor perspectives in medicine, (2019). 9(11): p. a036780.

21. Skelton, P.D., R.V. Stan, and B.W. Luikart, The role of PTEN in neurodevelopment. Molecular neuropsychiatry, (2019). 5(1): p. 60-71.

22. (!!! INVALID CITATION !!! [22-24]).

23. Cristofano, A.D., et al., Pten is essential for embryonic development and tumour suppression. Nature genetics, (1998). 19(4): p. 348-355.

24. Clipperton-Allen, A.E. and D.T. Page, Pten haploinsufficient mice show broad brain overgrowth but selective impairments in autism-relevant behavioral tests. Human molecular genetics, (2014). 23(13): p. 3490-3505.

25. Charney, D.S., E.J. Nestler, and M. Pamela Sklar, Charney \& Nestler's neurobiology of mental illness. (2017): Oxford University Press.

26. Courchesne, E., et al., Mapping early brain development in autism. Neuron, (2007). 56(2): p. 399-413.

27. Lee, H., et al., Constitutional mislocalization of Pten drives precocious maturation in oligodendrocytes and aberrant myelination in model of autism spectrum disorder. Translational psychiatry, (2019). 9(1): p. 1-12.

28. Tilot, A.K., et al., Germline disruption of Pten localization causes enhanced sex-dependent social motivation and increased glial production. Human molecular genetics, (2014). 23(12): p. 3212-3227.

29. Fricano-Kugler, C.J., et al., Nuclear excluded autism-associated phosphatase and tensin homolog mutations dysregulate neuronal growth. Biological psychiatry, (2018). 84(4): p. 265-277.

30. Yates, J.R.W., Tuberous sclerosis. European Journal of Human Genetics, (2006). 14(10): p. 1065-1073.

31. O'Callaghan, F.J., et al., Prevalence of tuberous sclerosis estimated by capture-recapture analysis. Lancet, (1998). 351(9114): p. 1490.

32. Northrup, H., D.A. Krueger, and G. International Tuberous Sclerosis Complex Consensus, Tuberous sclerosis complex diagnostic criteria update: recommendations of the 2012 Iinternational Tuberous Sclerosis Complex Consensus Conference. Pediatric neurology, (2013). 49(4): p. 243-254.

33. Islam, M.P., Tuberous Sclerosis Complex. Seminars in Pediatric Neurology, (2021). 37: p. 100875.

34. Sancak, O., et al., Mutational analysis of the TSC1 and TSC2 genes in a diagnostic setting: genotype - phenotype correlations and comparison of diagnostic DNA techniques in Tuberous Sclerosis Complex. European Journal of Human Genetics, (2005). 13(6): p. 731-741.

35. Valerio, N. and C. Paolo, Genetics and Molecular Biology of Tuberous Sclerosis Complex. Current Genomics, (2008). 9(7): p. 475-487.

36. Capal, J.K., et al., Influence of seizures on early development in tuberous sclerosis complex. Epilepsy \& Behavior, (2017). 70: p. 245-252.

37. Filosa, S., et al., Exploring the possible link between $\mathrm{MeCP} 2$ and oxidative stress in Rett syndrome. Free Radical Biology and Medicine, (2015). 88: p. 81-90.

38. Ramocki, M.B., et al., Autism and other neuropsychiatric symptoms are prevalent in individuals with MeCP2 duplication syndrome. Annals of Neurology, (2009). 66(6): p. 771-782.

39. Sandweiss, A.J., V.L. Brandt, and H.Y. Zoghbi, Advances in understanding of Rett syndrome and MECP2 duplication syndrome: prospects for future therapies. The Lancet Neurology, (2020). 19(8): p. 689-698.

40. Khalili Alashti, S., et al., Two novel mutations in the MECP2 gene in patients with Rett syndrome. Gene, (2020). 732: p. 144337.

41. Ciernia, A.V., et al., Early motor phenotype detection in a female mouse model of Rett syndrome is improved by cross-fostering. Human Molecular Genetics, (2017). 26(10): p. 1839.

42. Du, F., et al., Acute and crucial requirement for $\mathrm{MeCP} 2$ function upon transition from early to late adult stages of brain maturation. Human Molecular Genetics, (2016)(9): p. 1690-1702.

43. Chen, J., I. Alberts, and X. Li, Dysregulation of the IGF-I/PI3K/AKT/mTOR signaling pathway in autism spectrum disorders. International Journal of Developmental Neuroscience, (2014). 35: p. 35-41.

44. Stambolic, V., et al., Negative regulation of $\mathrm{PKB} /$ Akt-dependent cell survival by the tumor suppressor PTEN. Cell, (1998). 95(1): p. 29-39.

45. Darnell, J.C. and E. Klann, The translation of translational control by FMRP: therapeutic targets for FXS. Nature neuroscience, (2013). 16(11): p. 1530-1536.

46. Ehrhart, F., et al., Rett syndrome-biological pathways leading from MECP2 to disorder phenotypes. Orphanet journal of rare diseases, (2016). 11(1): p. 1-13. 